University of Zurich

Department of Economics

Working Paper Series

ISSN 1664-7041 (print)

ISSN1664-705X(online)

Working Paper No. 44

\title{
The Redistributive Effects of Monetary Policy
}

Olivier Ledoit

October 2011 


\title{
The Redistributive Effects of Monetary Policy
}

\author{
Olivier Ledoit* \\ Department of Economics \\ University of Zürich \\ olivier.ledoit@econ.uzh.ch
}

October 31, 2011

\begin{abstract}
We introduce a model of the economy as a social network. Two agents are linked to the extent that they transact with each other. This generates well-defined topological notions of location, neighborhood and closeness. We investigate the implications of our model for monetary economics. When a central bank increases the money supply, it must inject the money somewhere in the economy. We demonstrate that the agent closest to the location where money is injected is better off, and the one furthest is worse off. This redistribution channel is independent from the ones previously noted in the literature. Symmetrically, any decrease in the money supply redistributes purchasing power in the other direction. We also outline the testable implications of our model.
\end{abstract}

KEYWORDS: money, redistribution, policy, central bank, social network, topology

\section{Introduction}

This paper examines the redistributive role of monetary policy. In order to study redistribution, we must abandon the standard representative-agent framework and model heterogeneity between agents. There exists one well-known redistributive effect of monetary policy between heterogenous agents: expanding the money supply transfers real consumption from agents with larger cash

*Address for correspondence: Olivier Ledoit, Department of Economics, University of Zürich, Wilfriedstrasse 6, 8032 Zürich, Switzerland. The author wishes to thank the participants of the Spring 2009 London Quant Group conference, and especially Michael Wolf, as well as seminar participants at the University of Lausanne, the University of Zürich and Utah State University, for feedback on earlier versions of this paper. Any remaining errors are my own. 
balances to agents with smaller cash balances. This is because the real purchasing power of one unit of money decreases, and this decrease hurts more the agents with larger cash balances.

Our objective is to show that monetary expansion has another redistribution effect, completely independent from this one. To this end, we will present a model where all agents have the same cash endowment and the same degree of preference for money - but nonetheless, real consumption is redistributed.

The basic idea is that the economy is a social network. An agent is linked to certain other agents to the extent that he (or she) trades goods with them. But not everybody trades with everybody else equally. If a consumer opens the Yellow Pages, she will find that she has only ever dealt with a tiny fraction of the businesses listed. This network structure induces topological notions of location, neighborhood and distance onto the set of economic agents. It becomes legitimate to say that a certain event takes place "somewhere" in the economy, at a particular location and not anywhere else. $^{1}$

On this basis, when the head of the central bank decides to inject money into the economy, she must inject it somewhere. For either institutional and/or practical reasons, she cannot do it uniformly throughout the economy. Only a few institutions have direct dealings with the central bank. General members of the public have no access to the central bank, and must go through a chain of intermediaries. The money injected will certainly percolate through the social network that is the economy, but how? This is the question that the present paper addresses.

We take the simplest kind of network: a circle. Every agent on the circle is in close economic relationship with his two immediate neighbors (one neighbor next to him clockwise, and the other counterclockwise), and trades less intensely or not at all with the other agents. We use a pure exchange economy, where each agent is endowed with one unit of a certain consumption good (distinct from the others), and with the quantity of money $M$. In order to prove that our channel is independent from the well-known effect of the devaluation of cash balances, we must assume that all agents are endowed with the same quantity of money $M$. We also assume that all agents have the same degree of preference for money, within a money-in-utility framework.

The central bank increases the money supply by purchasing one particular good, say the good with which Agent 1 is endowed. We prove that this pushes up the price of good 1 more than the prices of the other consumption goods. This benefits Agent 1: he is strictly better off as a result of

\footnotetext{
${ }^{1}$ While this is beyond the scope of the paper, modelling the economy as a social network could prove useful in other areas of economics where heterogeneity also matters.
} 
being the only agent who deals directly with the central bank. We also show that the agent who is the furthest from the central bank, i.e. the one diametrically opposite from Agent 1 on the circle, is strictly worse off as a result. Therefore monetary expansion redistributes real consumption from the agent who is furthest from the central bank to the agent who is closest to the central bank.

The next section reviews the existing literature on this subject. Section 3 introduces our model of the economy as a social network. Section 4 solves for equilibrium prices. Section 5 shows that monetary policy has a redistributive effect even if all agents are endowed with the same amount of cash and have the same degree of preference for money. Section 6 outlines the testable implications of the model in terms of price inflation. Section 7 concludes. Appendix A contains the proof of one of the theorems.

\section{$2 \quad$ Literature Review}

To the best of our knowledge, the other articles on the redistributive effects of monetary policy go through the usual channel, in one way or another: the devaluation of cash balances. For example, in the overlapping generations model of Bhattacharya et al. (2005), monetary expansion redistributes real wealth from old agents (who hold large amounts of money) to young agents (who do not). For Palivos (2005), monetary expansion redistributes real wealth from altruistic agents (who hold large amounts of money because they want to bequeath it to their children) to selfish agents (who hold less money because they do not care about their children). Romer and Romer (1999) point out that inflation redistributes wealth from creditors to debtors. In the turnpike model of Shi (1999), monetary expansion redistributes real wealth from the rich (i.e. agents with a large endowment) to the poor (agents with a small endowment). To the contrary, Erosa and Ventura (2002) find that monetary expansion redistributes real wealth from the poor (who hold a large percentage of their wealth in cash) to the rich (who hold a large percentage of their wealth as capital instead of cash). Since all the agents in our model have the same cash balance and the same appetite for cash, our redistributive effect is completely independent from these.

This line of research belongs to the broader topic of money neutrality (see Lucas, 1996). The redistributive effect that we identify argues strongly for the non-neutrality of money. This question is also a subject of considerable interest in political economy. For example, Albanesi (2007) views the choice of monetary policy as the outcome of a political conflict over redistribution between low income households and high income households. Ireland (2005) argues that policy makers face a 
choice between engineering redistribution through monetary policy or through fiscal policy, and tries to discern which one of the two options is better.

\section{Model}

Agents Consider a pure exchange economy with an even number $N$ of agents: $N=2 n$ for some integer $n>1 .^{2}$ Each agent can be interpreted as the representative agent for a certain class of individuals. These $N$ agents are identical, except in one respect: the nature of the goods with which they are endowed and which they demand. The agents exchange money and $N$ distinct consumption goods. Agent $j$ is endowed with money and with good $j$ (for all $j=1, \ldots, N$ ). Let $M$ denote the quantity of money with which agent $j$ is endowed. Since we wish to make the agents as similar to one another as possible, $M$ is assumed to be the same across all agents. Furthermore, agent $j$ is endowed with one unit of good $j$. This assumption can be made without loss of generality, provided that we redefine the measurement unit of each good accordingly.

Objective Function Each agent is a price-taker with Cobb-Douglas utility function. The objective function of agent $j(j=1, \ldots, N)$ is:

$$
\begin{aligned}
& \max _{m_{j} ; x_{1 j}, \ldots, x_{n j}}\left(\frac{m_{j}}{\sum_{k=1}^{N} m_{k}}\right)^{\beta} \times \prod_{i=1}^{N} x_{i j}{ }^{\alpha_{i j}} \\
& \text { subject to: } \quad m_{j}+\sum_{i=1}^{n} p_{i} x_{i j} \leq M+p_{j}
\end{aligned}
$$

The price of good $i$ is $p_{i}$, and by convention the price of a unit of money is 1 . The budget available to agent $j$ is $M+p_{j}$. The quantity $m_{j}$ represents the amount of money demanded by agent $j$, and $x_{i j}$ represents the amount of good $i$ demanded by agent $j$ (for $i=1, \ldots, N$ ). As this is a one-period model, demand for money must be interpreted as reduced-form for holding a liquid asset that can easily be sold in order to finance any needs that may arise in the future. Normalizing the cash holding of agent $j$ by the sum of all cash holdings is necessary to avoid "money illusion": you cannot make everybody happier simply by multiplying everybody's cash holdings by a factor of ten. The Cobb-Douglas exponent $\beta$, which captures the intensity of demand for money, is assumed to be the same across all agents. We need $\beta>0$ and $\forall i=1, \ldots, N \quad \alpha_{i i}>0$. The other Cobb-Douglas

\footnotetext{
${ }^{2}$ The assumption that $N$ is even is not essential to our conclusions, but it facilitates the exposition of the model.
} 
exponents $\alpha_{i j}$ are all non-negative.

Circular Symmetry The agents are organized in a circle. See Figure 1 for an illustration. We

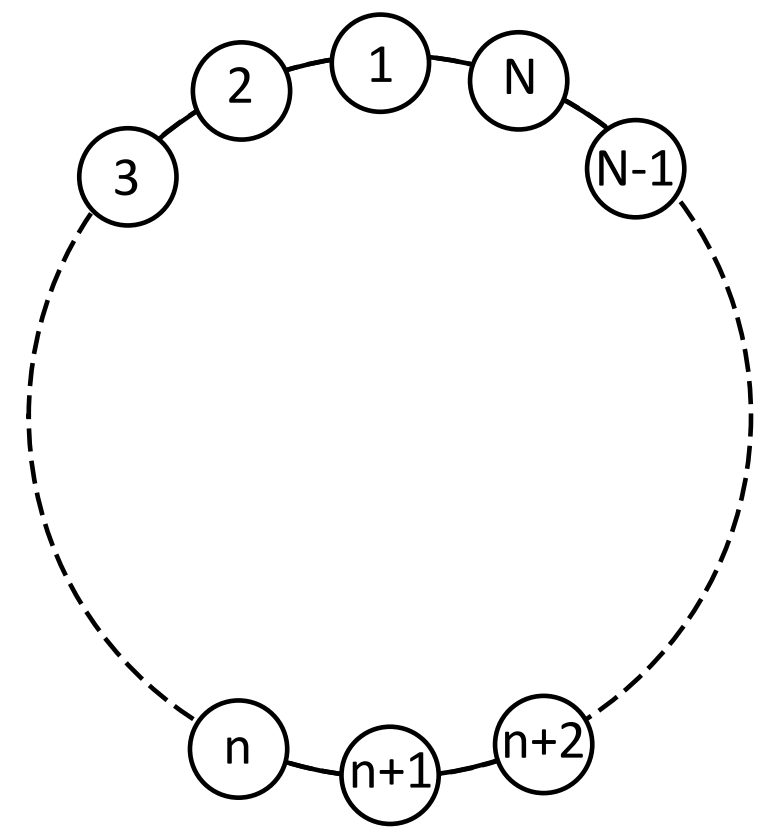

Figure 1: Graphical representation of the circular structure of the economy. Since the number of agents is $N=2 n$, the agent diametrically opposite Agent 1 is Agent $n+1$.

assume that the relationship of agent $i$ to agent $j$ depends only on the number of nodes that separates them: $\min (|i-j|,|N-i+j|)$. This assumption of circular symmetry implies a particular structure for the matrix $\mathbf{A}=\left(\alpha_{i j}\right)_{i, j=1, \ldots, N}$. There must exist $a_{0}>0$ and $N-1$ non-negative coefficients $a_{1}, \ldots, a_{N-1}$ such that:

$$
\mathbf{A}=\left(\alpha_{i j}\right)_{i, j=1, \ldots, N}=\left[\begin{array}{ccccc}
a_{0} & a_{1} & a_{2} & \cdots & a_{N-1} \\
a_{N-1} & a_{0} & a_{1} & \ddots & \vdots \\
a_{N-2} & a_{N-1} & a_{0} & \ddots & a_{2} \\
\vdots & \ddots & \ddots & \ddots & a_{1} \\
a_{1} & \ldots & a_{N-2} & a_{N-1} & a_{0}
\end{array}\right] .
$$

In mathematical terms, $\mathbf{A}$ is a circulant matrix. A circulant matrix is a matrix where each row vector is rotated one element to the right relative to the preceding row vector. Furthermore, $\mathbf{A}$ 
must be a symmetric circulant matrix, i.e. the following additional condition must be verified:

$$
\forall i=1, \ldots, N-1 \quad a_{N-i}=a_{i}
$$

Thus, the Cobb-Douglas exponent of the demand for good $i$ in the objective function of agent $j$ should only depend on the number of nodes that separates $i$ and $j$. This can be described as a rotation-invariant economy, since rotating the names of the agents by one or more notches, clockwise or counter-clockwise, does not modify any of the assumptions.

It will be convenient to denote by $S_{C}\left(z_{0}, z_{1}, \ldots, z_{n}\right)$ the symmetric circulant matrix whose first row vector is $\left[\begin{array}{llllllll}z_{0} & z_{1} & z_{2} \ldots z_{n-1} & z_{n} & z_{n-1} \ldots z_{2} & z_{1}\end{array}\right]$. With this notation, we can write: $\mathbf{A}=$ $S_{C}\left(a_{0}, a_{1}, \ldots, a_{n}\right)$.

An elegant way to rewrite the objective function is to adopt the convention that the indices for goods and agents are not elements of the set of all integers $\mathbb{Z}$, but of the cyclic group $\mathbb{Z} / N \mathbb{Z}$ generated by the number of agents $N$. Thus, in terms of indices, $i+N=i-N=i$, i.e. all the indices are interpreted modulo $N$. With this convention, Equation (1) becomes:

$$
\begin{aligned}
\max _{m_{j} ; x_{1 j}, \ldots, x_{N j}} & \left(\frac{m_{j}}{\sum_{k=1}^{N} m_{k}}\right)^{\beta} x_{j j}{ }^{a_{0}}\left[\prod_{k=1}^{n-1}\left(x_{j+k, j} x_{j-k, j}\right)^{a_{k}}\right] x_{j+n, j} a_{n} \\
& \text { subject to: } \quad m_{j}+\sum_{i=1}^{N} p_{i} x_{i j} \leq M+p_{j} .
\end{aligned}
$$

Neighborhood Effects We assume that agents have closer economic ties to their immediate neighbors than to distant neighbors. This is necessary in order to induce topological notions of location and closeness in the economy. It translates into the condition:

$$
a_{0} \geq a_{1}>a_{2} \geq a_{3} \geq \cdots \geq a_{n}
$$

In other words, agent $j$ has strictly more intense need for the goods in the initial endowment of agents $j-1$ and $j+1$ than for those of agents $j-2$ and $j+2$. In turn, agent $j$ has more intense (or the same) need for the goods in the initial endowment of agents $j-2$ and $j+2$ than for those of agents $j-3$ and $j+3$; and so forth. Of all the goods in the economy, the one for which agent $j$ has the least intense need is the one in the initial endowment of agent $j+n$, who is located diametrically opposite him on the circle.

The most simplistic case is: $a_{1}>0$ and $a_{2}=\cdots=a_{n / 2}=0$, i.e. an agent only transacts with 
the two neighbors on either side of him. But our results hold for the more general case described in Equation (4).

Central Bank The only institution that violates the rotation-invariance of the economy is the central bank. A central bank cannot have the same economic relationship to all the agents in the economy. Indeed, members of the general public are not usually allowed to deal with the central bank directly, but only through a sequence of intermediaries. One of the key roles of the head of the central bank is to control the money supply. She is authorized to inject money into the economy by buying certain assets and putting them on the central bank's balance sheet, or to reduce the amount of money outstanding by selling assets that were previously on its balance sheet. Either way, when the head of the central bank injects money, she must inject it somewhere, and when she reduces the money supply, she must take the money from somewhere. The notion of location implicit in the use of the word "somewhere" is well-defined in our economy because we have modelled the market as a social network, which generates proper topological notions of neighborhood and distance.

We assume that the head of the central bank injects money into the economy by buying one specific good at the prevailing market price, say good 1. Let $Q$ denote the quantity of money injected by the central bank. The quantity of good 1 that is taken away from the economy and placed onto the central bank's balance sheet is $Q / p_{1}$. $Q$ does not need to be strictly positive: if $Q<0$ then the central bank is reducing the money supply and selling $-Q / p_{1}$ units of good 1 from its balance sheet; finally, if $Q=0$ then the central bank is inactive, which is the only case where the rotation-invariance of the economy is respected.

\section{Equilibrium}

First-Order Conditions Let us start from the objective function of agent $j(j=1, \ldots, N)$ given in Equation (1). Note that $\sum_{k=1}^{N} m_{k}=N M+Q$, which is constant, so it can be dropped. Taking the logarithm of the Cobb-Douglas utility function yields:

$$
\begin{gathered}
\max _{m_{j} ; x_{1 j}, \ldots, x_{N j}} \beta \log \left(m_{j}\right)+\sum_{i=1}^{N} \alpha_{i j} \log \left(x_{i j}\right) \\
\text { subject to: } \quad m_{j}+\sum_{i=1}^{N} p_{i} x_{i j} \leq M+p_{j} .
\end{gathered}
$$


We can assume without loss of generality that:

$$
\beta+\sum_{i=1}^{N} \alpha_{i j}=1
$$

The Lagrangian is:

$$
\mathcal{L}=\beta \log \left(m_{j}\right)+\sum_{i=1}^{N} \alpha_{i j} \log \left(x_{i j}\right)-\lambda\left(m_{j}+\sum_{i=1}^{N} p_{i} x_{i j}-M-p_{j}\right)
$$

where $\lambda$ is the Lagrange multiplier. The first-order condition with respect to the demand for money $m_{j}$ is:

$$
\beta-\lambda m_{j}=0
$$

The first-order condition with respect to $x_{i j}$, the demand for good $i$, is:

$$
\alpha_{i j}-\lambda p_{i} x_{i j}=0 \quad \forall i=1, \ldots, N
$$

Substituting Equation (6) into Equation (7) yields:

$$
p_{i} x_{i j}=\frac{\alpha_{i j}}{\beta} m_{j} \quad \forall i=1, \ldots, N
$$

Market-Clearing Conditions The market-clearing conditions for goods 1 through $N$ are:

$$
\sum_{j=1}^{N} x_{i j}=1-\delta_{i 1} \frac{Q}{p_{1}} \quad \forall i=1, \ldots, N
$$

where $\delta$ denotes the Kronecker symbol, i.e. $\delta_{i 1}$ is equal to one if $i=1$ and zero otherwise. Multiplying both sides by the price yields:

$$
\sum_{j=1}^{N} p_{i} x_{i j}=p_{i}-\delta_{i 1} Q \quad \forall i=1, \ldots, N
$$

Using Equation (8), we get:

$$
\sum_{j=1}^{N} \frac{\alpha_{i j}}{\beta} m_{j}=p_{i}-\delta_{i 1} Q \quad \forall i=1, \ldots, N
$$


Remember that we have defined the matrix $\mathbf{A}=\left(\alpha_{i j}\right)_{i, j=1, \ldots, n}$. We can rewrite Equation (9) more synthetically in matrix form as:

$$
\beta^{-1} \mathbf{A}\left[\begin{array}{c}
m_{1} \\
\vdots \\
m_{N}
\end{array}\right]=\left[\begin{array}{c}
p_{1} \\
\vdots \\
p_{N}
\end{array}\right]-Q \mathbf{e}_{\mathbf{1}}
$$

where $\mathbf{e}_{\mathbf{1}}$ is a conformable vector with one in the first row and zeros everywhere else. ${ }^{3}$ Define $N$-dimensional column vectors for money demand $\mathbf{m}=\left[m_{1} \ldots m_{N}\right]^{\prime}$ and for prices $\mathbf{p}=\left[p_{1} \ldots p_{N}\right]^{\prime}$. Then we have:

$$
\beta^{-1} \mathbf{A m}=\mathbf{p}-Q \mathbf{e}_{\mathbf{1}}
$$

The market-clearing condition for money does not need to be examined as it will trivially be satisfied.

Budget Constraints Due to non-satiation, the budget constraint for a given agent $j(j=$ $1, \ldots, N)$ is an equality constraint:

$$
m_{j}+\sum_{i=1}^{N} p_{i} x_{i j}=M+p_{j} .
$$

Substituting Equation (8) into the budget constraint yields:

$$
m_{j}+\sum_{i=1}^{N} \frac{\alpha_{i j}}{\beta} m_{j}=M+p_{j}
$$

Thanks to Equation (5), this simplifies into:

$$
\beta^{-1} m_{j}=M+p_{j}
$$

If we vectorize this expression and multiply both sides from the left by the matrix $\mathbf{A}$, we obtain:

$$
\beta^{-1} \mathbf{A m}=M \mathbf{A} 1+\mathbf{A p}
$$

\footnotetext{
${ }^{3}$ Uppercase bold denotes matrices, and lowercase bold denotes vectors.
} 
where $\mathbf{1}$ denotes a conformable vector of ones. Since $\mathbf{A}$ is a symmetric circulant matrix, $\mathbf{1}$ is one of its eigenvectors:

$$
\mathbf{A} \mathbf{1}=\left(\sum_{j=1}^{N} \alpha_{1 j}\right) \mathbf{1}=\left(\sum_{i=0}^{N-1} a_{i}\right) \mathbf{1}=(1-\beta) \mathbf{1}
$$

So we get:

$$
\beta^{-1} \mathbf{A} \mathbf{m}=(1-\beta) M \mathbf{1}+\mathbf{A p} .
$$

Comparing with Equation (10) yields:

$$
\beta^{-1} \mathbf{A m}=\mathbf{p}-Q \mathbf{e}_{\mathbf{1}}=(1-\beta) M \mathbf{1}+\mathbf{A} \mathbf{p}
$$

After some algebraic manipulations, we finally obtain:

$$
(\mathbf{I}-\mathbf{A}) \mathbf{p}=(1-\beta) M \mathbf{1}+Q \mathbf{e}_{\mathbf{1}}
$$

where I denotes a conformable identity matrix. In order to go further, we need to prove a technical lemma.

Lemma 1 For any real square matrix $\mathbf{Z}$, let $\|\mathbf{Z}\|$ denote its spectral norm, which is defined as the square root of the largest eigenvalue of $\mathbf{Z} \mathbf{Z}^{\prime}$. Under the assumptions of Theorem $1,\|\mathbf{A}\|<1$.

Proof of Lemma 1 All circulant matrices are diagonalizable. Therefore, in order to prove the lemma, it is sufficient to prove that all the eigenvalues of $\mathbf{A}$ have modulus strictly lower than 1. Since $\mathbf{A}$ is a circulant matrix, its $j^{\text {th }}$ eigenvalue $(j=1, \ldots, n)$ is given by the well-known formula (see e.g. Gray, 2006, Chap. 3):

$$
l_{j}=\sum_{k=0}^{N-1} e^{-2 j k \pi i / N} a_{k}
$$

where $i=\sqrt{-1}$. Therefore its modulus is bounded by:

$\left|l_{j}\right|=\left|\sum_{k=0}^{N-1} e^{-2 j k \pi i / N} a_{k}\right| \leq \sum_{k=0}^{N-1}\left|e^{-2 j k \pi i / N} a_{k}\right| \leq \sum_{k=0}^{N-1}\left|e^{-2 j k \pi i / N}\right| \cdot\left|a_{k}\right| \leq \sum_{k=0}^{N-1}\left|a_{k}\right|=\sum_{k=0}^{N-1} a_{k}=1-\beta<1$.

This completes the proof of Lemma 1. 
Solution The bound $\|\mathbf{A}\|<1$ guarantees that the matrix $\mathbf{I}-\mathbf{A}$ is invertible. Therefore Equation (11) yields:

$$
\mathbf{p}=(1-\beta) M(\mathbf{I}-\mathbf{A})^{-1} \mathbf{1}+Q(\mathbf{I}-\mathbf{A})^{-1} \mathbf{e}_{\mathbf{1}}
$$

Define the matrix:

$$
\mathbf{\Lambda}=\left(\lambda_{i j}\right)_{i, j=1, \ldots, N}=(\mathbf{I}-\mathbf{A})^{-1}
$$

Since $\mathbf{1}$ is one of the eigenvectors of $\mathbf{A}$, it is also an eigenvector of $\mathbf{I}-\mathbf{A}$ and of $\boldsymbol{\Lambda}$. Therefore we have:

$$
\begin{aligned}
(\mathbf{I}-\mathbf{A}) \mathbf{1} & =\beta \mathbf{1} \\
\mathbf{\Lambda} \mathbf{1} & =\frac{1}{\beta} \mathbf{1} .
\end{aligned}
$$

This enables us to rewrite Equation (13) as:

$$
\forall i=1, \ldots, N \quad p_{i}=\frac{1-\beta}{\beta} M+\lambda_{i 1} Q
$$

We can summarize these results by the following theorem:

Theorem 1 If the following assumptions are satisfied:

(a) there are $N$ agents and $N$ real consumable goods (in addition to money), where $N$ is an even number: $N=2 n$ for some integer $n>1$;

(b) agent $j$ is endowed with the quantity of money $M>0$ and with one unit of good $j$;

(c) for all $j=1, \ldots, N$, agent $j$ has the objective function:

$$
\left(\frac{m_{j}}{\sum_{k=1}^{N} m_{k}}\right)^{\beta} x_{j j}^{a_{0}}\left[\prod_{k=1}^{n-1}\left(x_{j+k, j} x_{j-k, j}\right)^{a_{k}}\right] x_{j+n, j}^{a_{n}}
$$

where $m_{j}$ is his demand for money, and $x_{i j}$ is his demand for good $i(i=1, \ldots, N)$;

(d) $\beta>0$ and $a_{0} \geq a_{1}>a_{2} \geq a_{3} \geq \ldots \geq a_{n} \geq 0$;

(e) $Q$ is the change in the money supply that occurs as the central bank buys (if $Q \geq 0$ ) or sells (if $Q \leq 0$ ) good 1 at the market price; 
then the equilibrium price for good $i$ is:

$$
p_{i}=\frac{1-\beta}{\beta} M+\lambda_{i 1} Q \quad \forall i=1, \ldots, N
$$

where $\boldsymbol{\Lambda}=\left(\lambda_{i j}\right)_{i, j=1, \ldots, N}=(\mathbf{I}-\mathbf{A})^{-1}$ and $\mathbf{A}=S_{C}\left(a_{0}, a_{1}, \ldots, a_{n}\right)$.

The meaning of Equation (14) is intuitive: if the central bank does not intervene $(Q=0)$, then the rotation-invariance of the economy is respected and the prices of all real consumable goods are equal to the baseline price $\frac{1-\beta}{\beta} M$. However, as soon as the central bank intervenes $(Q \neq 0)$, rotation-invariance is violated, prices are distorted away from the baseline, and the size of the distortion is a linear function of the change in money supply $Q$.

\section{Comparative Statics}

In order to perform comparative statics for the influence of the change in the money supply $Q$ on equilibrium prices, we need to find out more about the $\lambda_{i 1}$ 's. This is achieved by the following theorem.

Theorem 2 Under the assumptions of Theorem 1,

$$
\begin{aligned}
\forall i=2, \ldots, N \quad & \lambda_{N+2-i, 1}=\lambda_{i 1} \\
& \lambda_{11}>\lambda_{21}>\ldots>\lambda_{n 1} \geq 0 .
\end{aligned}
$$

The proof of Theorem 2 is somewhat technical, so it is relegated to Appendix A.

Corollary 1 Under the assumptions of Theorem 1,

$$
\forall i=2, \ldots, N \quad p_{N+2-i}=p_{i}
$$

and:

(i) $Q=0 \Longrightarrow p_{1}=p_{2}=\cdots=p_{n+1}$;

(ii) $Q>0 \Longrightarrow p_{1}>p_{2}>\cdots>p_{n+1}$;

(iii) $Q<0 \Longrightarrow p_{1}<p_{2}<\cdots<p_{n+1}$. 
Proof of Corollary 1 This corollary follows immediately from Theorem 2 and Equation (14).

Corollary 2 Assume that the hypotheses of Theorem 1 are satisfied. Relative to the baseline case where the money supply is constant $(Q=0)$ :

(i) if $Q>0$ then Agent 1 is strictly better off and Agent $n+1$ is strictly worse off;

(ii) if $Q<0$ then Agent 1 is strictly worse off and Agent $n+1$ is strictly better off.

Proof of Corollary 2 If $Q>0(Q<0)$ then the prices of all the goods Agent 1 consumes go down (up) relative to the price of good 1, with which this agent is endowed. As a result, his budget constraint becomes strictly less (more) binding. Therefore he is strictly better (worse) off.

If $Q>0(Q<0)$ then the prices of all the goods Agent $n+1$ consumes go up (down) relative to the price of good $n+1$, with which this agent is endowed. As a result, his budget constraint becomes strictly more (less) binding. Therefore he is strictly worse (better) off.

For any other agent ( 2 through $n$ and $n+2$ through $N$ ), the impact of monetary intervention $(Q \neq 0)$ is ambiguous because, relative to the price of the good he is endowed with, some of the goods he consumes become more expensive, and others become cheaper.

\section{Testable Implications}

All testable implications of this theory hinge on the econometrician's ability to construct an index that measures the "Economic Distance from the Federal Reserve". Let us call it the EDFR index for short. We would have to assign an EDFR index to every price in a given dataset. This can cover consumer prices, producer prices, wages, or even asset prices. To the best of our knowledge, this type of endeavor has never been directly attempted in the literature. Yet it would only require the same kind of information that goes into building the input-output matrix of Leontief (1941), which models the structure of inter-industry relations.

Given an EDFR index, our model implies that prices will behave differently, depending on the index level they correspond to. There are testable implications in both the first and the second moment of price changes. In terms of the first moment, since the growth rate of the money supply is positive on average, the testable implication of the model is that prices associated with lower values of the EDFR index will rise faster on average than those with high EDFR indices. 
In terms of the second moment of price changes, the model offers a few different testable implications. The first is that prices with low (high) EDFR index are more (less) volatile. While the Federal Reserve injects money into the economy on average, it does not do so at a constant rate. Therefore these variations constitute an extra source of volatility, which is in addition to the other normal sources of volatility associated with supply and demand fluctuations. Federal Reserve-induced volatility affects low-EDFR prices more strongly than high-EDFR prices.

In addition, we would expect the correlation structure of prices to reflect this effect. Low-EDFR prices should be more correlated with each other than with high-EDFR prices. Furthermore, lowEDFR prices should be more correlated with each other than high-EDFR prices are correlated with each other. In other words, there should be an "EDFR factor" that can be extracted from the covariance matrix of price changes.

Finally, and this is more tentative, it might be possible to correlate changes in low-EDFR prices with the rate of growth in the money supply. However, in this test, the practical difficulties are great, because it is hard to know what measure of the money stock to use, and because there could be a long time lag that obfuscates the relationship.

\section{Conclusion}

This paper introduces a model of the economy as a social network. The basic point is that everybody does not trade equally with everybody else. This fact induces topological notions of distance, neighborhood and closeness on the set of economic agents. Our model even works in the least favorable case, where everybody trades with everybody, as long as every agent has stronger economic ties with his nearest neighbors than with the rest of the world. A direction for future research is to use the model of the economy as a social network in fields other than monetary economics where agent heterogeneity also matters.

One of the key roles of a central bank is to control the quantity of money. In order to do so, the central bank must either inject money into the financial system, or drain money from it. Necessarily, this money must be injected somewhere into the social network that is the economy, or drained from somewhere. The effects of monetary policy will percolate through the whole network, but not uniformly so. Whoever is closest to the location where money is injected (or drained) will be more strongly impacted. This is the effect that we have modelled mathematically.

Our model shows that whoever stands closest to (furthest from) the point where the central 
bank intervenes will benefit (suffer) from any increase in the money supply, and suffer (benefit) from any decrease in the money supply. Since the net cumulative effect of central bank interventions, over any sufficiently long period of time, is to increase the money supply, we can say that monetary policy redistributes consumption goods from the agents who are furthest from the central bank to those who are closest to the central bank.

Directions for future research also include compiling an approximate EDFR (Economic Distance from the Federal Reserve) index, so as to empirically examine the testable implications of our model. 


\section{A Proof of Theorem 2}

In order to prove the theorem, we must first prove 3 lemmas in succession. The most important of these lemmas is the first one.

Lemma 2 Let $\mathbf{X}=S_{C}\left(x_{0}, \ldots, x_{n}\right)$ and $\mathbf{Y}=S_{C}\left(y_{0}, \ldots, y_{n}\right)$ be two symmetric circulant matrices of dimension $N \times N$. Then $\mathbf{Z}=\mathbf{X Y}$ is also a symmetric circulant matrix: $\mathbf{Z}=S_{C}\left(z_{0}, \ldots, z_{n}\right)$. If $x_{0} \geq x_{1} \geq \cdots \geq x_{n} \geq 0$ and $y_{0} \geq y_{1} \geq \cdots \geq y_{n} \geq 0$ then $z_{0} \geq z_{1} \geq \cdots \geq z_{n} \geq 0$.

Proof of Lemma 2 All circulant matrices are diagonalizable on the same set of eigenvectors (see Gray, 2006, Chap. 3). It is well known that the product of two symmetric matrices that share the same set of eigenvectors is also a symmetric matrix. Furthermore, the product of two circulant matrices is a circulant matrix. Therefore $\mathbf{Z}=\mathbf{X Y}$ is a symmetric circulant matrix. By writing down the formula for the product of two matrices, we find that:

$$
\begin{aligned}
\forall k=0, \ldots, n \quad z_{k} & =\sum_{j=0}^{N-1} x_{j} y_{j-k} \\
z_{k+1}-z_{k} & =\sum_{j=0}^{N-1} x_{j}\left(y_{j-k-1}-y_{j-k}\right) .
\end{aligned}
$$

From Equation (A.1) it is obvious that when all the $x_{i}$ 's and all the $y_{i}$ 's are greater than or equal to zero, so are the $z_{i}$ 's. Now let us define: $y_{j}^{\prime}=y_{j}-y_{j-1}$ for all $j=0, \ldots, N-1$. It is easy to verify that $y_{N+1-j}^{\prime}=y_{1-j}^{\prime}=-y_{j}^{\prime}$. Furthermore, by the assumptions of Lemma $2, y_{j}^{\prime} \geq 0$ for $j \in\{0, n+1, n+2, \ldots, N-1\}$ and $y_{j}^{\prime} \leq 0$ for $j \in\{1,2, \ldots, n\}$. We can now rewrite Equation (A.2) more concisely as:

$$
\forall k=0, \ldots, n \quad z_{k+1}-z_{k}=-\sum_{j=0}^{N-1} x_{j} y_{j-k}^{\prime} .
$$

We shall prove that this quantity is nonpositive by considering two cases separately. We use the symbol \lfloor\rfloor to denote the floor function. 
First Case: $k \in\left\{0,1,2, \ldots,\left\lfloor\frac{n-1}{2}\right\rfloor\right\} \quad$ In this case, we can split the summation on the right-hand side of Equation (A.3) into:

$$
\begin{aligned}
z_{k+1}-z_{k}= & -\sum_{j=0}^{k} x_{j} y_{j-k}^{\prime}-\sum_{j=k+1}^{2 k+1} x_{j} y_{j-k}^{\prime}-\sum_{j=n}^{k+n} x_{j} y_{j-k}^{\prime} \\
& -\sum_{j=k+n+1}^{2 k+n+1} x_{j} y_{j-k}^{\prime}-\sum_{j=2 k+2}^{n-1} x_{j} y_{j-k}^{\prime}-\sum_{j=2 k+n+2}^{N-1} x_{j} y_{j-k}^{\prime} .
\end{aligned}
$$

Let us make the change of variable $i=2 k+1-j$ in the second summation, and the change of variable $i=2 k+N+1-j$ in the fourth and sixth summations. This yields:

$$
\begin{aligned}
z_{k+1}-z_{k}= & -\sum_{j=0}^{k} x_{j} y_{j-k}^{\prime}-\sum_{i=0}^{k} x_{2 k+1-i} y_{k+1-i}^{\prime}-\sum_{j=n}^{k+n} x_{j} y_{j-k}^{\prime} \\
& -\sum_{i=n}^{k+n} x_{2 k+N+1-i} y_{k+N+1-i}^{\prime}-\sum_{j=2 k+2}^{n-1} x_{j} y_{j-k}^{\prime}-\sum_{i=2 k+2}^{n-1} x_{2 k+N+1-i} y_{k+N+1-i}^{\prime} \\
= & -\sum_{j=0}^{k} x_{j} y_{j-k}^{\prime}+\sum_{i=0}^{k} x_{2 k+1-i} y_{i-k}^{\prime}-\sum_{j=n}^{k+n} x_{j} y_{j-k}^{\prime} \\
& +\sum_{i=n}^{k+n} x_{2 k+N+1-i} y_{i-k}^{\prime}-\sum_{j=2 k+2}^{n-1} x_{j} y_{j-k}^{\prime}+\sum_{i=2 k+2}^{n-1} x_{i-2 k-1} y_{i-k}^{\prime} \\
= & \sum_{j=0}^{k}\left(x_{2 k+1-j}-x_{j}\right) y_{j-k}^{\prime}+\sum_{j=n}^{k+n}\left(x_{2 k+N+1-j}-x_{j}\right) y_{j-k}^{\prime} \\
& +\sum_{j=2 k+2}^{n-1}\left(x_{j-2 k-1}-x_{j}\right) y_{j-k}^{\prime} .
\end{aligned}
$$

Let us consider these three summations separately:

- When $j \in\{0,1,2, \ldots, k\}$, we have:

$$
\begin{aligned}
& 0 \leq j \leq 2 k+1-j \leq n \quad \Longrightarrow \quad x_{2 k+1-j}-x_{j} \leq 0 \\
& \text { and } \quad 1-n \leq j-k \leq 0 \quad \Longrightarrow \quad y_{j-k}^{\prime} \geq 0
\end{aligned}
$$

therefore the $1^{\text {st }}$ summation is less than or equal to zero. 
- When $j \in\{n, n+1, \ldots, n+k\}$, we have:

$$
\begin{aligned}
n \leq j \leq 2 k+N+1-j \leq N & \Longrightarrow x_{2 k+N+1-j}-x_{j} \geq 0 \\
\text { and } \quad 1 \leq j-k \leq n & \Longrightarrow y_{j-k}^{\prime} \leq 0
\end{aligned}
$$

therefore the $2^{\text {nd }}$ summation is less than or equal to zero.

- When $j \in\{2 k+2,2 k+3, \ldots, n-1\}$, we have:

$$
\begin{aligned}
0 \leq j-2 k-1 \leq j \leq n & \Longrightarrow x_{j-2 k-1}-x_{j} \geq 0 \\
& \text { and } \quad 1 \leq j-k \leq n \quad \Longrightarrow \quad y_{j-k}^{\prime} \leq 0
\end{aligned}
$$

therefore the $3^{\text {rd }}$ summation is less than or equal to zero.

Together, these results establish that $z_{k+1}-z_{k} \leq 0$ for all $k \in\left\{0,1,2, \ldots,\left\lfloor\frac{n-1}{2}\right\rfloor\right\}$.

Second Case: $k \in\left\{\left\lfloor\frac{n-1}{2}\right\rfloor+1,\left\lfloor\frac{n-1}{2}\right\rfloor+2, \ldots, n-1\right\} \quad$ In this case, we can split the summation on the right-hand side of Equation (A.3) into:

$$
\begin{aligned}
z_{k+1}-z_{k}= & -\sum_{j=2 k-n+1}^{k} x_{j} y_{j-k}^{\prime}-\sum_{j=k+1}^{n} x_{j} y_{j-k}^{\prime}-\sum_{j=2 k+2}^{k+n} x_{j} y_{j-k}^{\prime} \\
& -\sum_{j=k+n+1}^{N-1} x_{j} y_{j-k}^{\prime}-\sum_{j=0}^{2 k-n} x_{j} y_{j-k}^{\prime}-\sum_{j=n+1}^{2 k+1} x_{j} y_{j-k}^{\prime} .
\end{aligned}
$$


Let us make the change of variable $i=2 k+1-j$ in the second and sixth summations, and the change of variable $i=2 k+N+1-j$ in the fourth summation. This yields:

$$
\begin{aligned}
z_{k+1}-z_{k}= & -\sum_{j=2 k-n+1}^{k} x_{j} y_{j-k}^{\prime}-\sum_{i=2 k-n+1}^{k} x_{2 k+1-i} y_{k+1-i}^{\prime}-\sum_{j=2 k+2}^{k+n} x_{j} y_{j-k}^{\prime} \\
& -\sum_{i=2 k+2}^{k+n} x_{2 k+N+1-i} y_{k+N+1-i}^{\prime}-\sum_{j=0}^{2 k-n} x_{j} y_{j-k}^{\prime}-\sum_{i=0}^{2 k-n} x_{2 k+1-i} y_{k+1-i}^{\prime} \\
= & -\sum_{j=2 k-n+1}^{k} x_{j} y_{j-k}^{\prime}+\sum_{i=2 k-n+1}^{k} x_{2 k+1-i} y_{i-k}^{\prime}-\sum_{j=2 k+2}^{k+n} x_{j} y_{j-k}^{\prime} \\
& +\sum_{i=2 k+2}^{k+n} x_{2 k+N+1-i} y_{i-k}^{\prime}-\sum_{j=0}^{2 k-n} x_{j} y_{j-k}^{\prime}+\sum_{i=0}^{2 k-n} x_{N-2 k-1+i} y_{i-k}^{\prime} \\
& \sum_{j=}^{k}\left(x_{2 k+1-j}-x_{j}\right) y_{j-k}^{\prime}+\sum_{j=2 k+2}^{k+n}\left(x_{2 k+N+1-j}-x_{j}\right) y_{j-k}^{\prime} \\
& +\sum_{j=0}^{2 k-n}\left(x_{N-2 k-1+j}-x_{j}\right) y_{j-k}^{\prime} .
\end{aligned}
$$

Let us consider these three summations separately:

- When $j \in\{2 k-n+1,2 k-n+2, \ldots, k\}$, we have:

$$
\begin{aligned}
& 0 \leq j \leq 2 k+1-j \leq n \quad \Longrightarrow \quad x_{2 k+1-j}-x_{j} \leq 0 \\
& \text { and } \quad 1-n \leq j-k \leq 0 \quad \Longrightarrow \quad y_{j-k}^{\prime} \geq 0
\end{aligned}
$$

therefore the $1^{\text {st }}$ summation is less than or equal to zero.

- When $j \in\{2 k+2,2 k+3, \ldots, k+n\}$, we have:

$$
\begin{aligned}
n \leq j \leq 2 k+N+1-j \leq n & \Longrightarrow x_{2 k+N+1-j}-x_{j} \geq 0 \\
\text { and } \quad 1 \leq j-k \leq n & \Longrightarrow y_{j-k}^{\prime} \leq 0
\end{aligned}
$$

therefore the $2^{\text {nd }}$ summation is less than or equal to zero. 
- When $j \in\{0,1,2, \ldots, 2 k-n\}$, we have:

$$
\begin{aligned}
0 \leq j \leq N-2 k-1+j \leq n & \Longrightarrow x_{N-2 k-1+j}-x_{j} \leq 0 \\
& \text { and } \quad 1-n \leq j-k \leq 0 \quad \Longrightarrow \quad y_{j-k}^{\prime} \geq 0
\end{aligned}
$$

therefore the $3^{\text {rd }}$ summation is less than or equal to zero.

Together, these results establish that $z_{k+1} \leq z_{k}$ for all $k \in\left\{\left\lfloor\frac{n-1}{2}\right\rfloor+1,\left\lfloor\frac{n-1}{2}\right\rfloor+2, \ldots, n-1\right\}$.

Bringing together the two cases, we conclude that $z_{0} \geq z_{1} \geq \cdots \geq z_{n}$. This completes the proof of Lemma 2.

Lemma 3 Let $\mathbf{D}$ be a symmetric circulant matrix: $\mathbf{D}=S_{C}\left(d_{0}, d_{1}, \ldots, d_{n}\right)$, where $d_{0} \geq d_{1} \geq$ $\ldots \geq d_{n} \geq 0$. Then for all $m \in \mathbb{N} \quad \mathbf{D}^{m}$ is also symmetric circulant matrix: $\mathbf{D}^{m}=S_{C}\left(d_{0 m}, d_{1 m}\right.$, $\left.\ldots, d_{n m}\right)$. Furthermore, we have: $d_{0 m} \geq d_{1 m} \geq \ldots \geq d_{n m} \geq 0$.

Proof of Lemma 3 We shall prove the lemma by induction. For $m=0, \mathbf{D}^{0}=\mathbf{I}$, so the statement is trivially true. Now let us suppose that the statement is true for some power $m \in \mathbb{N}$. We have the decomposition: $\mathbf{D}^{m+1}=\mathbf{D}^{m} \mathbf{D}$. Notice that the hypotheses of Lemma 2 are verified when we take $\mathbf{X}=\mathbf{D}^{m}$ and $\mathbf{Y}=\mathbf{D}$. So Lemma 2 implies that the statement is true for the power $m+1$. Therefore, by induction, the statement is true for all $m \in \mathbb{N}$.

Lemma 4 Under the assumptions of Lemma 3, if $d_{1}>d_{2}$ then for all $m=0, \ldots, n-1 d_{m m}>$ $d_{m+1, m}$.

Proof of Lemma 4 We shall prove this lemma by induction. Since $\mathbf{D}^{0}=\mathbf{I}$, we have $d_{00}=1$ and $d_{01}=0$. Therefore the statement is true for $m=0$. Now let us suppose that the statement is true for some $m \in\{0, \ldots, n-2\}$. Consider the decomposition: $\mathbf{D}^{m+1}=\mathbf{D}^{m} \mathbf{D}$. The hypotheses of Lemma 2 are verified when we take $\mathbf{Z}=\mathbf{D}^{m+1}, \mathbf{X}=\mathbf{D}^{m}$ and $\mathbf{Y}=\mathbf{D}$. From Equations (A.4-A.5) in the proof of Lemma 2, we know that:

$$
\forall k=0, \ldots, n-1 \quad z_{k+1}-z_{k} \leq \sum_{j=\max (0,2 k-n+1)}^{k}\left(x_{2 k+1-j}-x_{j}\right) y_{j-k}^{\prime}
$$

where all the terms in the summation are less than or equal to zero. Taking $k=m+1$ and $j=m$, we obtain:

$$
z_{m+2}-z_{m+1} \leq\left(x_{m+3}-x_{m}\right) y_{-1}^{\prime} .
$$


Since

$$
\begin{aligned}
& x_{m+3}-x_{m} \leq x_{m+1}-x_{m}<0 \\
& \text { and } y_{-1}^{\prime}=y_{2}^{\prime}=y_{2}-y_{1}<0
\end{aligned}
$$

by assumption, it follows that: $z_{m+2}-z_{m+1}<0$, i.e. $d_{m+2, m+1}-d_{m+1, m+1}<0$. Therefore the statement is true for $m+1$. By induction, this means that it is true for all $m=0, \ldots, n-1$. This completes the proof of Lemma 4.

Proof of the Theorem We are now ready to complete the proof of Theorem 2. Since the matrix A satifies the assumptions of Lemma 3, we know that for all $m \in \mathbb{N} \quad \mathbf{A}^{m}$ is a symmetric circulant matrix: $\mathbf{A}^{m}=S_{C}\left(a_{0 m}, a_{1 m}, \ldots, a_{n m}\right)$, and that: $a_{0 m} \geq a_{1 m} \geq \ldots \geq a_{n m} \geq 0$.

The bound $\|\mathbf{A}\|<1$ established in Lemma 1 guarantees that the matrix $\mathbf{I}-\mathbf{A}$ is invertible, i.e. that $\boldsymbol{\Lambda}=(\mathbf{I}-\mathbf{A})^{-1}$ exists. Since $\mathbf{A}$ is a symmetric circulant matrix, $\mathbf{I}-\mathbf{A}$ is also a circulant matrix, and so is $\boldsymbol{\Lambda}=(\mathbf{I}-\mathbf{A})^{-1}$. Thus, we can write: $\boldsymbol{\Lambda}=S_{C}\left(l_{0}, l_{1}, \ldots, l_{n}\right)$. The layout of a symmetric circulant matrix is such that:

$$
\begin{aligned}
\forall i=1, \ldots, n+1 & \lambda_{i 1}=l_{i-1} \\
\forall i=n+2, \ldots, N & \lambda_{i 1}=l_{N+1-i} .
\end{aligned}
$$

This implies that $\forall i=2, \ldots, N \quad \lambda_{N+2-i}=\lambda_{i}$, which was one of the assertions of Theorem 2 that needed to be proven.

Thanks to Lemma 1, we know that the series $\sum_{m=0}^{\infty} \mathbf{A}^{m}$ is convergent. Standard arguments (see e.g. Szidarovszky and Molnár, 2002, pp. 375-376) show that the sum of this series is: $\sum_{m=0}^{\infty} \mathbf{A}^{m}=$ $(\mathbf{I}-\mathbf{A})^{-1}=\boldsymbol{\Lambda}$. In particular, the entries of the matrix $\boldsymbol{\Lambda}$ satisfy:

$$
\forall j=0, \ldots, n \quad l_{j}=\sum_{m=0}^{\infty} a_{j m} .
$$

Therefore they inherit their ordering from the $a_{j m}$ 's, and we have:

$$
l_{0} \geq l_{1} \geq \ldots \geq l_{n} \geq 0
$$


The fact that these inequalities are actually strict:

$$
l_{0}>l_{1}>\ldots>l_{n}
$$

is a straightforward application of Lemma 4, given the assumption $a_{1}>a_{2}$. Therefore:

$$
\lambda_{11}>\lambda_{21}>\ldots>\lambda_{n+1,1} \geq 0 .
$$




\section{References}

Albanesi, S., 2007. Inflation and inequality. Journal of Monetary Economics 54, 1088-1114.

Bhattacharya, J., Haslag, J., Martin, A., 2005. Heterogeneity, redistribution, and the Friedman rule. International Economic Review 46, 437-454.

Erosa, A., Ventura, G., 2002. On inflation as a regressive consumption tax. Journal of Monetary Economics 49, 761-795.

Gray, R., 2006. Toeplitz and Circulant Matrices: A Review. Now Publishers Inc.

Ireland, P., 2005. Heterogeneity and redistribution: by monetary or fiscal means? International Economic Review 46, 455-463.

Leontief, W., 1941. The Structure of the American Economy, 1919-1929. Harvard University Press, Cambridge, MA.

Lucas, R., 1996. Nobel lecture: Monetary neutrality. Journal of Political Economy 104, 661-682.

Palivos, T., 2005. Optimal monetary policy with heterogeneous agents: a case for inflation. Oxford Economic Papers 57, 34-50.

Romer, C., Romer, D., 1999. Monetary policy and the well-being of the poor. Economic Review 1st quarter. Kansas City Federal Reserve.

Shi, S., 1999. Money, capital, and redistributive effects of monetary policies. Journal of Economic Dynamics and Control 23, 565-590.

Szidarovszky, F., Molnár, S., 2002. Introduction to Matrix Theory: with applications to business and economics. World Scientific. 